\title{
Regulation of telomere length in Drosophila
}

\author{
Radmila Capkova Frydrychova ${ }^{a}$, Harald Biessmann ${ }^{b}$, and James M. Mason ${ }^{a}$ \\ aLaboratory of Molecular Genetics, National Institute of Environmental Health Sciences, Research Triangle \\ Park, NC (USA) \\ bDevelopmental Biology Center, University of California, Irvine, CA (USA)
}

\begin{abstract}
Telomeres in all organisms must perform the same vital functions to ensure cell viability: to act as a protective chromosome cap that distinguishes natural chromosome ends from DNA double strand breaks, and to balance the loss of DNA from the chromosome end due incomplete DNA replication. Most eukaryotes rely on a specialized reverse transcriptase, telomerase, to generate short repeats at the chromosome end to maintain chromosome length. Drosophila, however, uses retrotransposons that target telomeres. Transposition of these elements may be controlled by small RNAs and spreading of silent chromatin from the telomere associated sequence, both of which limit the retrotransposon expression level. Proteins binding to the retrotransposon array, such as HP1 an PROD, may also modulate transcription. It is not clear, however, that simply increasing transcript levels of the telomeric retrotransposons is sufficient to increase transposition. The chromosome cap may control the ability of the telomere-specific elements to attach to chromosome ends. As in other organisms, chromosomes can be elongated by gene conversion. Although the mechanism is not known, HP1, a component of the cap, and the Ku proteins are key components in this pathway.
\end{abstract}

\section{Introduction}

Telomeres are specialized nucleoprotein structures at the ends of linear chromosomes that perform several functions essential to the life of a cell. First, they preserve chromosomal integrity by protection of chromosome ends from DNA damage response mechanisms that would otherwise recognize chromosome ends as double strand breaks (Ciapponi and Cenci, this issue). This function is often referred to as the chromosome cap. Telomeres also solve the end replication problem, i.e. the inability of DNA polymerase to replicate a linear DNA molecule fully to its very end. As a result of incomplete replication, it has been predicted that chromosome ends will shorten during each cell cycle (Watson, 1972; Olovnikov, 1973). The most widespread mechanism used to solve the end-replication problem is the function of telomerase, a ribonucleoprotein that adds telomeric repeats onto existing telomeres (this issue). Telomerase, however, is not the only way to extend telomeres, as gene conversion has also been demonstrated in a number of organisms, including those using telomerase, and telomerespecific retrotransposons have been observed in Drosophila (Mason and Biessmann, 1995), and alternative lengthening of telomeres (Bryan et al., 1995; Bryan et al., 1997) by recombination has been found in many sarcomas that do not express telomerase (Henson et al., 2005). It has been proposed (de Lange, 2004) in fact that telomeres in early eukaryotes may have been maintained by a form of recombination dependent replication before the advent of telomerase.

Corresponding author: Dr. James M. Mason, Laboratory of Molecular Genetics, National Institute of Environmental Health Sciences, P.O. Box 12233, Research Triangle Park, NC 27709-2233, telephone: 1-919-541-4483; fax: 1-919-541-7593, email: masonj@niehs.nih.gov. 
In Drosophila, genetic assays for the activity of reporter genes at or near the end of truncated chromosomes revealed two mechanisms of telomere maintenance, terminal gene conversion and targeted transposition of telomeric retrotransposons. In this review, we discuss factors that participate in the regulation the two mechanisms. Transposition of the telomere-specific transposons may be modulated by transcriptional activity of these elements, which in turn is regulated by rasiRNA. Control of terminal gene conversion is still poorly understood in Drosophila, but a protein complex at the chromosome end seems to be important in modulating accessibility of the telomere for both terminal gene conversion and targeting retroelement RNA to chromosome ends. At least some components of this protein complex also participate in the capping function.

\section{Telomeric retrotransposons}

Most chromosome ends in Drosophila carry an array of telomere specific non-long terminal repeat (LTR) retrotransposons, HeT-A, TART and TAHRE (collectively abbreviated here as HTT) (Fig. 1A) that are present in multiple copies at chromosome ends. Transposition of these retroelements may occur via target-primed reverse transcription (Fig. 2), which attaches these elements to the chromosome ends by their 3' oligo(A) tails. This transposition does not depend on a specific DNA at the target site and, coupled with terminal erosion, produces head-to-tail arrays of mixed complete and 5' truncated elements at the ends of Drosophila chromosomes (Fig. 1B) (Levis et al., 1993;Walter et al., 1995;Abad et al., 2004b;Pardue et al., 2005). TART and TAHRE each have two open reading frames (ORFs). ORF2, which encodes a reverse transcriptase (RT), is not present in $H e T-A$. This leads to the prediction that the RT required for transposition of $H e T-A$ is produced in trans from an unknown source (Biessmann et al., 1992a;Biessmann et al., 1994). HeT-A and TAHRE share a sequence similarity as well as a pattern of transcription, leading to the hypothesis that TAHRE is the source of reverse transcriptase enzymatic activity for $\mathrm{HeT}-\mathrm{A}$ transposition (Abad et al., 2004a;Shpiz et al., 2007). ORF1, which is found in all three elements, encodes a GAG-like protein that binds to the retroelement RNA and transports it back into the nucleus (Rashkova et al., 2002a) and, at least for HeT-A, associates with chromosome ends (Rashkova et al., 2002b). This association may be involved in targeting transposition to chromosome ends. Each telomeric retroelement has an unusually long $3^{\prime}$ untranslated region (UTR) that, in HeT-A and TAHRE, contains a promoter transcribing the downstream element (Danilevskaya et al., 1997;Capkova Frydrychova et al., 2007; Shpiz et al., 2007). There are several promoters on TART making transcripts from both strands of the element (Danilevskaya et al., 1999; Maxwell et al., 2006).

Not all telomeres in Drosophila include a HTT array. Terminal deficiency chromosomes lacking the HTT array have been found in natural populations (Mechler et al., 1985) and in laboratory stocks (Mason et al., 2004). Terminal deficiencies can also be induced in the presence of a mutator mutation in the gene mu2 (Mason et al., 1984; Mason et al., 1997), or when breaks occur in the vicinity of an existing telomere (Levis, 1989; Tower et al., 1993). While lacking the HTT array, these chromosomes are stable over many generations without inducing cell-cycle-arrest, which suggests that these chromosomes possess a chromosome cap that is formed independently of telomeric DNA sequence (Biessmann and Mason, 1988, 2003).

In telomeres that carry a HTT array the array length varies widely. Abad et al. (Abad et al., 2004b) described telomere length in individual chromosomes of one stock in the range of 26-147 kb. Other stocks with shorter (Walter et al., 1995; Golubovsky et al., 2001) and longer (Melnikova and Georgiev, 2002; Siriaco et al., 2002) HTT arrays have been described. Incomplete replication and oxidative stress are considered to be reasons of telomere shortening (Epel et al., 2004; Richter and von Zglinicki, 2007). In Drosophila, telomere shortening at 
terminally deficient chromosomes was estimated at a constant rate of about 75 bp per fly generation (Biessmann and Mason, 1988; Levis, 1989; Mikhailovsky et al., 1999).

As Drosophila telomeres differ from those of other eukaryotes, different mechanisms may be used to maintain telomere length within acceptable limits. Addition of HTT sequence onto chromosome ends has been measured at $\sim 1 \%$ per generation in a wild type stock, with and average length for the added fragment of $6 \mathrm{~kb}$, just enough to balance terminal erosion (Biessmann et al., 1992a). Different stocks, however, may vary in the frequency at which these additions occur by two orders of magnitude in either direction (Golubovsky et al., 2001; Savitsky et al., 2002; Savitsky et al., 2006). On the other hand, distinct mechanisms may operate to limit HTT array length. While extremely long telomeres do not increase lifespan in flies, they may be eliminated from the population due to their negative effect on fecundity and fertility (Walter et al., 2007).

\section{HP1 and PROD modulate transcription of telomeric retroelements}

Several loci have been identified in Drosophila that are required to prevent end-to-end chromosome fusions, thus indicating their role in chromosome capping (Savitsky et al., 2002; Perrini et al., 2004; Cenci et al., 2005); Ciapponi and Cenci, this issue), although the only protein that has been identified to play a role in both capping and telomere elongation is heterochromatin protein 1, HP1 (Cenci et al., 2005). HP1 is a conserved chromosomal protein showing, on polytene chromosomes of Drosophila, an association with pericentric heterochromatin, telomeres and specific euchromatic regions (Fanti et al., 2003). Through association of HP1 with histone H3 methylated at Lys 9 (H3MeK9), heterochromatin can spread into transgenes inserted into pericentric regions, leading to a repression of the transcriptional activity of these transgenes (Li et al., 2002; Danzer and Wallrath, 2004; Hediger and Gasser, 2006). Despite its role in heterochromatin formation and its repressive effect on transgenes, however, it is noteworthy that HP1 works also as a transcriptional activator of heterochromatic and some euchromatic genes (Piacentini et al., 2003; Cryderman et al., 2005). Mutations in the gene encoding HP1 ( $\mathrm{Su}$ (var)205), however, lead to a 100 fold increase in HeT-A transcripts (Perrini et al., 2004).

Telomere elongation by addition of HTT elements to the chromosome end can occur by two distinct mechanisms, terminal gene conversion or targeted transposition (Fig. 2). As shown in Figure 3, new HTT attachments onto a chromosome end can be monitored genetically by activation of a terminally located white ( $w$ ) or yellow (y) gene by the $3^{\prime}$ promoter of a HeT-A element (Golubovsky et al., 2001;Savitsky et al., 2002). The effects of reduced HP1 levels on telomere elongation were studied by monitoring the frequency of retroelement additions to a terminally deficient $X$ chromosome (Savitsky et al., 2002). Telomere elongation is sensitive to HP1 concentration, as $\mathrm{Su}$ (var)205 mutations lead to more than 100 fold higher HeT-A/TART attachments compared to $\mathrm{Su}(\mathrm{var}) 205^{+}$flies. This increased frequency of retroelement attachments in $\mathrm{Su}(\mathrm{var}) 205$ mutants results from both increased retroelement transposition and terminal gene conversion, and is associated with extremely long telomeres after several generations (Savitsky et al., 2002). The gene conversion events do not use sequences on the homologue or another chromosome, but depend on the presence of homologous sequence on the same chromosome in what is essentially a tandem array. Thus, terminal gene conversion by this mechanism requires a structure that at least superficially resembles a t-loop (Greider, 1999), and one possible function of HP1 might be to prevent this configuration. The rate of DNA attachment to chromosome ends in $\mathrm{Su}$ (var)205 mutants was shown to occur in germline and somatic cells without significant differences among the alleles tested, including the $\mathrm{Su}$ (var) $205^{02}$ allele, which allows mutant HP1 localization at telomeres and does not affect telomere capping. As the $\mathrm{Su}$ (var) $205^{02}$ mutation is a one amino acid substitution in the chromodomain, it was concluded that the chromodomain, although dispensable for chromosome capping, is 
required for repression of both transcriptional activity of telomeric retroelements and telomere elongation (Perrini et al., 2004).

Telomeres in Drosophila display some overlap between HP1 and H3MeK9. While Andreyeva et al. (Andreyeva et al., 2005) using immunostaining of polytene chromosomes found HP1 in the cap and histone $\mathrm{H} 3$ trimethylated at Lys 9 (H3Me3K9) in the HTT array outside of the cap, with no overlap, we found using chromatin immunoprecipitation relatively high levels of both $\mathrm{HP} 1$ and histone $\mathrm{H} 3$ dimethylated at Lys $9(\mathrm{H} 3 \mathrm{Me} 2 \mathrm{~K} 9)$ at the subtelomeric telomere associated sequence (TAS) and in the HTT array outside of the cap region (Capkova Frydrychova et al., 2008). The presence of $\mathrm{H} 3 \mathrm{MeK} 9$ at telomeres and the repression of the transcriptional activity of the telomeric retrotransposons both depend on the presence of a functional HP1

chromodomain (Perrini et al., 2004). Thus, it is conceivable that the heterochromatin formed by the interaction of HP1with H3MeK9 is necessary for control of telomere elongation. It is important to note, however, that despite the predicted function of H3MeK9 in Drosophila telomere elongation, the histone methyltransferase responsible for methylation of $\mathrm{H} 3 \mathrm{~K} 9$ at telomeres is not encoded by either $E(z)$ or $S u(v a r) 3-9$ (Perrini et al., 2004). Another crucial finding for understanding telomere behavior in Drosophila is that there are two separate modes of HP1 binding to telomeres: telomere capping depends on direct binding of HP1 to terminal DNA mediated by the hinge domain, while the transcriptional control of telomeric retrotransposons depends on the interaction of the HP1 chromodomain with H3MeK9.

Another gene affecting the transcriptional activity of telomeric retroelements is prod. The PROD protein localizes strongly to the centric heterochromatin of the second and third chromosomes, to telomeres and to $>400$ euchromatic sites (Török et al., 1997). In the HTT array, PROD binds to a region just upstream of the HeT-A promoter (Török et al., 2007). Heterozygous prod mutants exhibit elevated levels of $H e T-A$ transcript in ovaries, suggesting that prod may be involved in the control of telomere elongation as a repressor of HeT-A transcription, although it is without a demonstrated role in telomere capping. However, these elevated HeT-A transcript levels do not cause telomere growth (Török et al., 2007), suggesting that the terminal protein cap may be a predominant control point for telomere elongation and that increased HTT transcripts are not sufficient for elevated HTT transposition.

\section{RNAi and germline control of HTT elements}

Telomere elongation by transposition of HTT elements involves an RNA intermediate, which serves as messenger for the translation of GAG and RT proteins and as a template for reverse transcription (Fig. 2). Thus, controlling HTT RNA levels, especially in the germline, where new transpositions to the chromosome ends will be passed on to the next generation, might be an important regulatory mechanism for telomere elongation. One of the factors controlling HTT RNA production is repeat-associated small interfering RNA (rasiRNA). In general, small RNAs downregulate gene expression through an RNA-interference (RNAi) pathway, protect genome stability and suppress the spread of selfish retrotransposons and other repetitive sequences (Aravin et al., 2007; O'Donnell and Boeke, 2007). The rasiRNA pathway requires members of the Piwi subfamily of Argonaute proteins, PIWI, AUB and AGO3, which bind directly to small RNAs and use these as guides for identification and, through their slicer activity, cleavage of their targets (Aravin et al., 2007;Brennecke et al., 2007). These three proteins are expressed in male and female germ cells; additionally PIWI is found in the somatic cells that are in close contact with germ cells (Aravin et al., 2007). PIWI, AUB and AGO3 associate with a specific class of small RNAs (piwi-interacting RNAs, piRNAs) ranging in length from 23-29 nt. Surveys of rasiRNAs have identified piRNAs derived from HeT-A, TAHRE and TART among many from other retrotransposons in Drosophila ovaries (Saito et al., 2006;Brennecke et al., 2007). The rasiRNAs from HeT-A and TART were also found in early embryos (Aravin et al., 2003). PIWI binds mostly sense piRNAs from HeT-A and 
TAHRE, but antisense rasiRNAs from TART; AUB is associated with antisense rasiRNAs of all three, and AGO3 binds mainly sense rasiRNAs of all three HTT elements (Brennecke et al., 2007).

These data indicate that transcript levels of the telomeric retrotransposons are probably under the control of the rasiRNA pathway. RNA levels of HTT retroelements, as well as other retroelements were greatly elevated in the germline of mutants for genes involved in the rasiRNA pathway and the putative RNA helicase genes spn-E and armi, whereas mutations in genes of the siRNA pathway had no effect (Sarot et al., 2004; Vagin et al., 2004; Kalmykova et al., 2005; Savitsky et al., 2006; Vagin et al., 2006; Brennecke et al., 2007; Pelisson et al., 2007; Shpiz et al., 2007; Desset et al., 2008). Mutations in the cuff gene also resulted in increased levels of HeT-A and TART transcripts in ovaries, but the role of cuff in the rasiRNA pathway, if any, is not known (Chen et al., 2007). An indication of how HeT-A transcript levels might be regulated by the rasiRNA pathway in the germline comes from the observation that the reduced abundance of rasiRNA in ovaries but not in somatic cells of $s p n-E$ mutants is accompanied by a change in the chromatin structure of genomic HeT-A elements and other telomeric retrotransposons (Klenov et al., 2007). HeT-A chromatin may become more transcriptionally active in spn-E mutants, as indicated by an increase in the active chromatin mark histone $\mathrm{H} 3$ dimethylated at Lys 4, a decrease in the inactive chromatin marks $\mathrm{H} 3 \mathrm{Me} 2 \mathrm{~K} 9$ and $\mathrm{H} 3 \mathrm{Me} 3 \mathrm{~K} 9$, and a reduction of HP1 associated with the promoter and the coding region of HeT-A (Klenov et al., 2007).

Do these elevated transcript levels of the telomeric retrotransposons actually lead to increased transpositions to the chromosome ends and thus to elongated HTT arrays at the telomeres? As described above, transcript levels for HeT-A and TART are elevated in ovaries of spn-E and $a u b$ mutants. Going a step further, Savitsky et al. (Savitsky et al., 2006) tested transposition of these elements to a broken $X$ chromosome and found a $~ 100$-fold increased frequency of terminal retrotransposon attachment in $s p n-E$ and a smaller increase in $a u b$ heterozygotes compared to wild type, although the control had an unusually low transposition frequency. The rates of terminal attachments in heterozygous $s p n-E^{1}$ and $a u b^{Q C 42}$ were very close to the $1 \%$ per generation expected in order to maintaina constant chromosome length (Biessmann et al., 1992a). The majority were TART transpositions with only few that were HeT-A, which is unexpected because transcript levels of both elements are elevated. In homozygous spn-E females seven independent $H e T$-A but no TART transpositions were observed, consistent with the 35-fold increase in HeT-A and 7-fold in TART transcript levels. The total genomic copy numbers of HeT-A and TART, a measure for telomere length, were not increased in spn-E and $a u b$ stocks, suggesting again that even elevated transcript levels are not sufficient for chromosome elongation. We have confirmed that HTT arrays do not grow long in $s p n-E$ and $a u b$ heterozygotes, even when they have been maintained in stock for several years (R. Capkova Frydrychova and J. M. Mason unpublished).

\section{RNAi and silencing by TAS}

TAS is the source of heterochromatic silencing at Drosophila telomeres, and its influence represses transgenes inserted into it, a phenomenon termed telomeric position effect, TPE (recently reviewed in (Mason et al., 2008)). Silencing also spreads from TAS distally into the HTT array, where it may downregulate retrotransposon promoters and thus repress their transcriptional activity (Capkova Frydrychova et al., 2007). Therefore, the heterochromatic state of TAS may also be a factor in telomeric length control.

TAS carries marks of inactive chromatin, especially Polycomb group proteins (Boivin et al., 2003; Andreyeva et al., 2005). Silencing at TAS may be mediated through RNAi pathways, as rasiRNA transcripts from 2L TAS were detected in 2-4 hr-old embryos (Aravin et al., 
2003) and also from $X$ TAS, 4R TAS and 3R TAS (Brennecke et al., 2007). This latter RNA has been mapped to the distal end of the 3R TAS (Yin and Lin, 2007). Its levels are reduced in piwi mutants, reflecting the observations that euchromatic histone modifications in the $3 \mathrm{R}$ TAS region from which the piRNA is transcribed are decreased and heterochromatic marks are increased in these mutants. Thus, PIWI promotes euchromatic chromatin features in TAS, consistent with the observation that loss of piwi function acts as enhancer of TPE when tested with the partially silenced $P\left\{w^{+}, r y^{+}\right\} A 4-4$ (hereafter A4-4) transgene, which is located in $3 \mathrm{R}$ TAS just proximal to the piRNA coding sequence (Yin and Lin, 2007). This element causes overexpression of this rasiRNA in wild type flies and in piwi mutants and rescues the germ cell defects of piwi (Smulders-Srinivasan and Lin, 2003). One interpretation of these results postulates that PIWI binds to the distal end of $3 R$ TAS allowing some read-through transcription from the retrotransposon promoters a short distance into TAS. When the $20 \mathrm{~kb}$ A4-4 element is present proximal to the piRNA region, it shields this part of TAS from the bulk of the $3 R$ TAS, rendering it less heterochromatic and more amenable to transcription from the adjacent retrotransposon promoters (Yin and Lin, 2007).

The rasiRNA pathway also mediates telomeric trans-silencing in the female germline, in which a transgene or transposon inserted into $X \mathrm{~L}$ TAS can repress expression of a homologous insertion located elsewhere in the genome (Josse et al., 2007). This trans-silencing in some ways resembles $\mathrm{P}$ cytotype, a mechanism for repressing $P$ element activity, in which a $P$ element in $X \mathrm{~L}$ TAS represses other $P$ elements throughout the genome (Ronsseray et al., 1991; Marin et al., 2000; Niemi et al., 2004; Simmons et al., 2004) via an RNAi pathway sensitive to mutations in $a u b$ but not piwi (Simmons et al., 2007).

In summary, it seems that piRNA is acting differently in TAS and the HTT array. In TAS PIWI promotes open chromatin and increased transcription of elements inserted into TAS, while in the HTT array PIWI promotes closed chromatin and downregulates expression of the HTT retrotransposons. At the same time, TAS plays a role in HTT expression, at least in its immediate vicinity.

\section{Telomere elongation by terminal conversion}

Monitoring of $X$ chromosomes that terminate in the yellow ( $y$ ) gene region allowed two types of elongation events to be observed, retroelement transposition and terminal gene conversion (Biessmann et al., 1990; Biessmann et al., 1992a; Biessmann et al., 1992b; Mikhailovsky et al., 1999). Terminal gene conversion that transfers sequence to a broken chromosome end was analyzed in the presence of a full-length homologous $X$ chromosome in females. In this system terminal deficiency chromosomes were monitored for elongation by re-attachment of previously deleted $y$ enhancers via gene conversion from the homologous $X$ chromosome to the broken end (Mikhailovsky et al., 1999). The average length of the conversion track was estimated as $2.7 \mathrm{~kb}$. Alternatively, by taking advantage of the promoter activity in the 3' UTR of HeT-A (Danilevskaya et al., 1997), Kahn et al. (Kahn et al., 2000) studied HeT-A

transposition to a terminally deficient $X$ chromosome that had a break in the $y$ promoter region and thus loss of promoter activity (Fig. 3B). After HeT-A transposition to such chromosomes, the HeT-A promoter initiates transcription of the downstream $y \mathrm{ORF}$, resulting in restoration of pigmentation in bristles. To evaluate the respective contributions of HeT-A transposition and terminal conversion, Kahn et al. (Kahn et al., 2000) examined both length and sequence of attachments to truncated $X$ chromosomes. They found that from eighteen independent elongation events the majority were new HeT-A transpositions and only four to six involved terminal gene conversion. Thus, transposition may be the predominant mechanism for extension of broken chromosome ends in Drosophila, although in the presence of homologous sequences elsewhere in the genome, terminal gene conversion can also contribute. 
In these experiments the chromosome end was within the unique sequences of the $y$ gene, which served either as site for the hybridization of specific DNA probes or as genetic marker for phenotypic changes occurring due to the attachment of new sequences to the end. It is generally assumed, however, that these two elongation mechanisms also take place at the end of natural chromosome ends that terminate in long retrotransposon arrays (Fig. 2). Such extensions of the HTT array cannot be monitored molecularly or genetically because they add retrotransposons to an already existing array of the same retrotransposons. If, however, an imbalance exists between elongation and shortening due to genetic factors, telomeres will either grow or shrink. This can be measured by determining the overall HTT array length or the genomic copy number of HTT retroelements.

A dominant mutation in the $E(t c)$ gene significantly enhances terminal conversion but has no effect on terminal retrotransposition frequency (Melnikova and Georgiev, 2002). A similar phenotype of long telomeres has been observed in the presence of another dominant mutation, Tel (Siriaco et al., 2002), which maps to the same genetic region as $E(t c)$. The mechanism of $T e l$ action has not been elucidated, but HeT-A copy numbers at telomeres are increased about seven-fold, TART and TAHRE somewhat less (Siriaco et al., 2002; Walter et al., 2007).

\section{The cap and telomere elongation}

A number of factors may affect telomere length, and extended HTT arrays are a good indicator of an imbalance in the telomere elongation process, regardless of mechanism. As discussed above, extended HTT arrays have been observed in the presence of heterozygous $\mathrm{Su}$ (var)205 mutations (Perrini et al., 2004; Török et al., 2007), which cause an increase in both telomeretargeted retrotransposition and telomeric gene conversion (Savitsky et al., 2002). A significant increase in the frequency of HTT retrotranspositions and terminal conversions is also caused by partial inactivation of the Ku70/Ku80 complex, a component of the nonhomologous end joining (NHEJ) pathway (Melnikova et al., 2005). As reduction in Ku concentration does not increase the level of HeT-A transcription, it is suggested that Ku controls accessibility of the chromosome end to elongation events. While these results with HP1 and the Ku70/Ku80 complex suggest that the telomere cap regulates chromosome elongation, it is worth noting that flies heterozygous for $c a v$, the gene encoding the capping component HOAP, do not show increased genomic copy numbers of telomeric retrotransposons (H. Biessmann, unpublished) or increased HeT-A transcript levels (Capkova Frydrychova et al., 2008).

Together, these observations indicate that some, but not all, components of the chromosome cap are important in regulating telomere length. Reducing their efficacy in protecting the end causes telomeres to lengthen by either retrotransposition or terminal gene conversion. Thus, the cap may serve a dual function with regard to telomere elongation. On one hand, it protects the chromosome end from elongation events; on the other hand, it appears to be a docking site for the retrotransposon-GAG complex, which generates a crucial intermediate for the reverse transcription of the retrotransposon transcript as depicted in Fig. 2. This notion is supported by the observation that GFP-tagged HeT-A GAG proteins accumulate in nuclear foci (Rashkova et al., 2002a), which may represent their association with existing telomeres at chromosome ends as the majority of these foci co-localize with the capping protein HOAP (Rashkova et al., 2002b). The sites on the HeT-A GAG protein necessary for targeting telomeres are separate from the nuclear localization signal and distributed along the protein (Rashkova et al., 2003). The tagged TART GAG, however, can enter the nucleus, but only associates with telomeric foci when co-expressed with HeT-A GAG (Rashkova et al., 2002b). Using mammalian and yeast telomeres as guides (Hug and Lingner, 2006;Blasco, 2007), one might suggest that the Drosophila telomeric cap may also be regulated by epigenetic protein modifications, which in turn could determine accessibility of the chromosome end to the Drosophila-specific elongation 
mechanisms. These epigenetic modifications and their effect on the Drosophila cap have yet to be determined.

\section{Conclusion}

Although there are some similarities in the protein composition of the HTT array and the chromosome cap, the general picture that emerges from the published data is that Drosophila has a clear separation between the retrotransposon system that solves the end-replication problem and the protein complexes used for capping. This separation may be rooted in the evolution of the Drosophila telomere. It has been proposed that the Drosophila telomeric retrotransposons are derived from an ancestral element that was originally recruited to replace telomerase (Louis, 2002; Villasante et al., 2007). Such domestication of selfish transposable elements, which would bring the element under control of the host genome (Jensen et al., 1999; Miller et al., 1999), often involves RNAi-based regulation of transcript abundance as discussed in this review. Thus, regulation of telomere length in Drosophila involves modulation of retroelement transcript levels via rasiRNA, as well by chromatin structure emanating from TAS, and by binding of specific proteins, such as PROD, to the retrotransposon promoter.

As in species with telomerase, chromosome stability is controlled by a terminal protein complex. In Drosophila the components of the complex may be distinguished genetically revealing the dual function of the terminal cap. Proteins that participate in the chromosome cap, for example HP1 and HOAP, distinguish telomeres from broken chromosome ends, while an overlapping set of telomeric proteins, including HP1 and Ku, modulate accessibility of the chromosome end to elongation events, including terminal gene conversion. Thus, while in some respects fly telomeres differ from those of other eukaryotes, Drosophila may add a fresh perspective and better understanding of telomere biology in other organisms.

\section{Acknowledgments}

We thank Shay Covo and Gil Dos Santo for critically reading the manuscript.

This work was supported by U.S. Public Health Services grant GM-56729 and by the Intramural Research Program of the NIH, National Institute of Environmental Health Sciences, project ES021054

\section{References}

Abad JP, de Pablos B, Osoegawa K, de Jong PJ, Martin-Gallardo A, et al. TAHRE, a novel telomeric Retrotransposon from Drosophila melanogaster, reveals the origin of Drosophila telomeres. Mol Biol Evol 2004a;21:1620-1624. [PubMed: 15175413]

Abad JP, de Pablos B, Osoegawa K, de Jong PJ, Martin-Gallardo A, et al. Genomic analysis of Drosophila melanogaster telomeres: Full-length copies of HeT-A and TART elements at telomeres. Mol Biol Evol 2004b;21:1613-1619. [PubMed: 15163766]

Andreyeva EN, Belyaeva ES, Semeshin VF, Polkholkova GV, Zhimulev IF. Three distinct chromatin domains in telomere ends of polytene chromosomes in Drosophila melanogaster Tel mutants. J Cell Sci 2005;118:5465-5477. [PubMed: 16278293]

Aravin AA, Hannon GJ, Brennecke J. The Piwi-piRNA pathway provides an adaptive defense in the transposon arms race. Science 2007;318:761-764. [PubMed: 17975059]

Aravin AA, Lagos-Quintana M, Yalcin A, Zavolan M, Marks D, et al. The small RNA profile during Drosophila melanogaster development. Dev Cell 2003;5:337-350. [PubMed: 12919683]

Biessmann H, Mason JM. Progressive loss of DNA sequences from terminal chromosome deficiencies in Drosophila melanogaster. EMBO J 1988;7:1081-1086. [PubMed: 2841109]

Biessmann H, Mason JM. Telomerase-independent mechanisms of telomere elongation. Cell Mol Life Sci 2003;60:2325-2333. [PubMed: 14625679] 
Biessmann H, Champion LE, O'Hair M, Ikenaga K, Kasravi B, et al. Frequent transpositions of Drosophila melanogaster HeT-A transposable elements to receding chromosome ends. EMBO J 1992a;11:4459-4469. [PubMed: 1330538]

Biessmann H, Kasravi B, Bui T, Fujiwara G, Champion LE, et al. Comparison of two active HeT-A retroposons of Drosophila melanogaster. Chromosoma 1994;103:90-98. [PubMed: 8055715]

Biessmann H, Mason JM, Ferry K, d'Hulst M, Valgeirsdottir K, et al. Addition of telomere-associated HeT DNA sequences "heals" broken chromosome ends in Drosophila. Cell 1990;61:663-673. [PubMed: 2111731]

Biessmann H, Valgeirsdottir K, Lofsky A, Chin C, Ginther B, et al. HeT-A, a transposable element specifically involved in healing broken chromosome ends in Drosophila melanogaster. Mol Cell Biol 1992b;12:3910-3918. [PubMed: 1324409]

Blasco MA. The epigenetic regulation of mammalian telomeres. Nat Rev Genet 2007;8:299-309. [PubMed: 17363977]

Boivin A, Gally C, Netter S, Anxolabehere D, Ronsseray S. Telomeric associated sequences of Drosophila recruit Polycomb-group proteins in vivo and can induce pairing-sensitive repression. Genetics 2003;164:195-208. [PubMed: 12750332]

Brennecke J, Aravin AA, Stark A, Dus M, Kellis M, et al. Discrete small RNA-generating loci as master regulators of transposon activity in Drosophila. Cell 2007;128:1089-1103. [PubMed: 17346786]

Bryan TM, Englezou A, Gupta J, Bacchetti S, Reddel RR. Telomere elongation in immortal human cells without detectable telomerase activity. EMBO Journal 1995;14:4240-4248. [PubMed: 7556065]

Bryan TM, Englezou A, Dallapozza L, Dunham MA, Reddel RR. Evidence for an alternative mechanism for maintaining telomere length in human tumors and tumor-derived cell lines. Nature Medicine 1997;3:1271-1274.

Capkova Frydrychova R, Mason JM, Archer TK. HP1 regulates transcription activity of HeT-A elements located along the retrotransposon array in Drosophila telomeres. Genetics. 2008in press

Capkova Frydrychova R, Biessmann H, Konev AY, Golubovsky MD, Johnson J, et al. Transcriptional activity of the telomeric retrotransposon $\mathrm{HeT}$-A in Drosophila melanogaster is stimulated as a consequence of subterminal deficiencies at homologous and nonhomologous telomeres. Mol Cell Biol 2007;27:4991-5001. [PubMed: 17470550]

Cenci G, Ciapponi L, Gatti M. The mechanism of telomere protection: a comparison between Drosophila and humans. Chromosoma 2005;114:135-145. [PubMed: 16012858]

Chen Y, Pane A, Schüpbach T. Cutoff and aubergine mutations result in retrotransposon upregulation and checkpoint activation in Drosophila. Curr Biol 2007;17:637-642. [PubMed: 17363252]

Cryderman DE, Grade SK, Li Y, Fanti L, Pimpinelli S, et al. Role of Drosophila HP1 in euchromatic gene expression. Dev Dyn 2005;232:767-774. [PubMed: 15704177]

Danilevskaya ON, Arkhipova IR, Traverse KL, Pardue ML. Promoting in tandem: the promoter for telomere transposon HeT-A and implications for the evolution of retroviral LTRs. Cell 1997;88:647655. [PubMed: 9054504]

Danilevskaya ON, Traverse KL, Hogan NC, Debaryshe PG, Pardue ML. The two Drosophila telomeric transposable elements have very different patterns of transcription. Mol Cell Biol 1999;19:873-881. [PubMed: 9858610]

Danzer JR, Wallrath LL. Mechanisms of HP1-mediated gene silencing in Drosophila. Development 2004;131:3571-3580. [PubMed: 15215206]

de Lange T. T-loops and the origin of telomeres. Nature Reviews Molecular Cell Biology 2004;5:323329.

Desset S, Buchon N, Meignin C, Coiffet M, Vaury C. In Drosophila melanogaster the COM Locus Directs the Somatic Silencing of Two Retrotransposons through both Piwi-Dependent and -Independent Pathways. PLoS ONE 2008;3:e1526. [PubMed: 18253480]

Epel ES, Blackburn EH, Lin J, Dhabhar FS, Adler NE, et al. Accelerated telomere shortening in response to life stress. Proc Natl Acad Sci USA 2004;101:17312-17315. [PubMed: 15574496]

Fanti L, Berloco M, Piacentini L, Pimpinelli S. Chromosomal distribution of Heterochromatin Protein 1 (HP1) in Drosophila: a cytological map of euchromatic HP1 binding sites. Genetica 2003;117:135147. [PubMed: 12723693] 
Golubovsky MD, Konev AY, Walter MF, Biessmann H, Mason JM. Terminal retrotransposons activate a subtelomeric white transgene at the 2L telomere in Drosophila. Genetics 2001;158:1111-1123. [PubMed: 11454760]

Greider CW. Telomeres do D-loop-T-loop. Cell 1999;97:419-422. [PubMed: 10338204]

Hediger F, Gasser SM. Heterochromatin protein 1: don't judge the book by its cover. Curr Opin Genet Dev 2006;16:143-150. [PubMed: 16503133]

Henson JD, Hannay JA, McCarthy SW, Royds JA, Yeager TR, et al. A robust assay for alternative lengthening of telomeres in tumors shows the significance of alternative lengthening of telomeres in sarcomas and astrocytomas. Clinical Cancer Research 2005;11:217-225. [PubMed: 15671549]

Hug N, Lingner J. Telomere length homeostasis. Chromosoma 2006;115:413-425. [PubMed: 16741708]

Jensen S, Gassama MP, Heidmann T. Taming of transposable elements by homology-dependent gene silencing. Nat Genet 1999;21:209-212. [PubMed: 9988275]

Josse T, Teysset L, Todeschini AL, Sidor CM, Anxolabehere D, et al. Telomeric Trans-silencing: An epigenetic repression combining RNA silencing and heterochromatin formation. Plos Genet 2007;3:1633-1643. [PubMed: 17941712]

Kahn T, Savitsky M, Georgiev P. Attachment of HeT-A sequences to chromosomal termini in Drosophila melanogaster may occur by different mechanisms. Mol Cell Biol 2000;20:7634-7642. [PubMed: 11003659]

Kalmykova AI, Klenov MS, Gvozdev VA. Argonaute protein PIWI controls mobilization of retrotransposons in the Drosophila male germline. Nucleic Acids Res 2005;33:2052-2059. [PubMed: 15817569]

Klenov MS, Lavrov SA, Stolyarenko AD, Ryazansky SS, Aravin AA, et al. Repeat-associated siRNAs cause chromatin silencing of retrotransposons in the Drosophila melanogaster germline. Nucleic Acids Res 2007;35:5430-5438. [PubMed: 17702759]

Levis RW. Viable deletions of a telomere from a Drosophila chromosome. Cell 1989;58:791-801. [PubMed: 2548737]

Levis RW, Ganesan R, Houtchens K, Tolar LA, Sheen FM. Transposons in place of telomeric repeats at a Drosophila telomere. Cell 1993;75:1083-1093. [PubMed: 8261510]

Li YH, Kirschmann DA, Wallrath LL. Does heterochromatin protein 1 always follow code? Proc Natl Acad Sci USA 2002;99:16462-16469. [PubMed: 12151603]

Louis, E. Are Drosophila telomeres an exception or the rule?. 2002. http://genomebiologycom/2002/3/10/reviews/0007 3:reviews0007.0001-0007.0006

Marin L, Lehmann M, Nouaud D, Hassan I, Anxolabéhère D, et al. P-element repression in Drosophila melanogaster by a naturally occurring defective telomeric $P$ copy. Genetics 2000;155:1841-1854. [PubMed: 10924479]

Mason JM, Biessmann H. The unusual telomeres of Drosophila. Trends Genet 1995;11:58-62. [PubMed: 7716808]

Mason JM, Strobel E, Green MM. mu-2: mutator gene in Drosophila that potentiates the induction of terminal deficiencies. Proc Natl Acad Sci USA 1984;81:6090-6094. [PubMed: 6435123]

Mason JM, Champion LE, Hook G. Germ-line effects of a mutator, mu2, in Drosophila melanogaster. Genetics 1997;146:1381-1397. [PubMed: 9258681]

Mason JM, Ransom J, Konev AY. A deficiency screen for dominant suppressors of telomeric silencing in Drosophila. Genetics 2004;168:1353-1370. [PubMed: 15579690]

Mason JM, Frydrychova RC, Biessmann H. Drosophila telomeres: an exception providing new insights. Bioessays 2008;30:25-37. [PubMed: 18081009]

Maxwell PH, Belote JM, Levis RW. Identification of multiple transcription initiation, polyadenylation, and splice sites in the Drosophila melanogaster TART family of telomeric retrotransposons. Nucleic Acids Res 2006;34:5498-5507. [PubMed: 17020919]

Mechler BM, McGinnis W, Gehring WJ. Molecular cloning of lethal(2)giant larvae, a recessive oncogene of Drosophila melanogaster. EMBO J 1985;4:1551-1557. [PubMed: 3928370]

Melnikova L, Georgiev P. Enhancer of terminal gene conversion, a new mutation in Drosophila melanogaster that induces telomere elongation by gene conversion. Genetics 2002;162:1301-1312. [PubMed: 12454074] 
Melnikova L, Biessmann H, Georgiev P. The Ku protein complex is involved in length regulation of Drosophila telomeres. Genetics 2005;170:221-235. [PubMed: 15781709]

Mikhailovsky S, Belenkaya T, Georgiev P. Broken chromosomal ends can be elongated by conversion in Drosophila melanogaster. Chromosoma 1999;108:114-120. [PubMed: 10382073]

Miller WJ, McDonald JF, Nouaud D, Anxolabehere D. Molecular domestication--more than a sporadic episode in evolution. Genetica 1999;107:197-207. [PubMed: 10952213]

Niemi JB, Raymond JD, Patrek R, Simmons MJ. Establishment and maintenance of the P cytotype associated with telomeric $P$ elements in Drosophila melanogaster. Genetics 2004;166:255-264. [PubMed: 15020423]

O’Donnell KA, Boeke JD. Mighty Piwis defend the germline against genome intruders. Cell 2007;129:37-44. [PubMed: 17418784]

Olovnikov AM. A theory of marginotomy. J Theor Biol 1973;41:181-190. [PubMed: 4754905]

Pardue ML, Rashkova S, Casacuberta E, DeBaryshe PG, George JA, et al. Two retrotransposons maintain telomeres in Drosophila. Chromosome Res 2005;13:443-453. [PubMed: 16132810]

Pelisson A, Sarot E, Payen-Groschene G, Bucheton A. A novel repeat-associated small interfering RNAmediated silencing pathway downregulates complementary sense gypsy transcripts in somatic cells of the Drosophila ovary. J Virol 2007;81:1951-1960. [PubMed: 17135323]

Perrini B, Piacentini L, Fanti L, Altieri F, Chichiarelli S, et al. HP1 controls telomere capping, telomere elongation, and telomere silencing by two different mechanisms in Drosophila. Mol Cell 2004;15:467-476. [PubMed: 15304225]

Piacentini L, Fanti L, Berloco M, Perrini B, Pimpinelli S. Heterochromatin protein 1 (HP1) is associated with induced gene expression in Drosophila euchromatin. Journal of Cell Biology 2003;161:707714. [PubMed: 12756231]

Rashkova S, Karam SE, Pardue ML. Element-specific localization of Drosophila retrotransposon Gag proteins occurs in both nucleus and cytoplasm. Proc Natl Acad Sci USA 2002a;99:3621-3626. [PubMed: 11891280]

Rashkova S, Athanasiadis A, Pardue ML. Intracellular targeting of Gag proteins of the Drosophila telomeric retrotransposons. J Virol 2003;77:6376-6384. [PubMed: 12743295]

Rashkova S, Karam SE, Kellum R, Pardue ML. Gag proteins of the two Drosophila telomeric retrotransposons are targeted to chromosome ends. J Cell Biol 2002b;159:397-402. [PubMed: 12417578]

Richter T, von Zglinicki T. A continuous correlation between oxidative stress and telomere shortening in fibroblasts. Experimental Gerontology 2007;42:1039-1042. [PubMed: 17869047]

Ronsseray S, Lehmann M, Anxolabehere D. The maternally inherited regulation of P elements in Drosophila melanogaster can be elicited by two P copies at cytological site $1 \mathrm{~A}$ on the $\mathrm{X}$ chromosome. Genetics 1991;129:501-512. [PubMed: 1660427]

Saito K, Nishida KM, Mori T, Kawamura Y, Miyoshi K, et al. Specific association of Piwi with rasiRNAs derived from retrotransposon and heterochromatic regions in the Drosophila genome. Gene Dev 2006;20:2214-2222. [PubMed: 16882972]

Sarot E, Payen-Groschene G, Bucheton A, Pelisson A. Evidence for a piwi-dependent RNA silencing of the gypsy endogenous retrovirus by the Drosophila melanogaster flamenco gene. Genetics 2004;166:1313-1321. [PubMed: 15082550]

Savitsky M, Kravchuk O, Melnikova L, Georgiev P. Heterochromatin protein 1 is involved in control of telomere elongation in Drosophila melanogaster. Mol Cell Biol 2002;22:3204-3218. [PubMed: 11940677]

Savitsky M, Kwon D, Georgiev P, Kalmykova A, Gvozdev V. Telomere elongation is under the control of the RNAi-based mechanism in the Drosophila germline. Gene Dev 2006;20:345-354. [PubMed: 16452506]

Shpiz S, Kwon D, Uneva A, Kim M, Klenov M, et al. Characterization of Drosophila telomeric retroelement TAHRE: Transcription, transpositions, and RNAi-based regulation of expression. Mol Biol Evol 2007;24:2535-2545. [PubMed: 17890237]

Simmons MJ, Raymond JD, Niemi JB, Stuart JR, Merriman PJ. The P cytotype in Drosophila melanogaster: A maternally transmitted regulatory state of the germ line associated with telomeric $P$ elements. Genetics 2004;166:243-254. [PubMed: 15020422] 
Simmons MJ, Ryzek DF, Lamour C, Goodman JW, Kummer NE, et al. Cytotype regulation by telomeric $P$ elements in Drosophila melanogaster: Evidence for involvement of an RNA interference gene. Genetics 2007;176:1945-1955. [PubMed: 17603126]

Siriaco GM, Cenci G, Haoudi A, Champion LE, Zhou C, et al. Telomere elongation (Tel), a new mutation in Drosophila melanogaster that produces long telomeres. Genetics 2002;160:235-245. [PubMed: 11805059]

Smulders-Srinivasan TK, Lin H. Screens for piwi suppressors in Drosophila identify dosage-dependent regulators of germline stem cell division. Genetics 2003;165:1971-1991. [PubMed: 14704180]

Török T, Harvie PD, Buratovich M, Bryant PJ. The product of proliferation disrupter is concentrated at centromeres and required for mitotic chromosome condensation and cell proliferation in Drosophila. Gene Dev 1997;11:213-225. [PubMed: 9009204]

Török T, Benitez C, Takacs S, Biessmann H. The protein encoded by the gene proliferation disrupter ( prod) is associated with the telomeric retrotransposon array in Drosophila melanogaster. Chromosoma 2007;116:185-195. [PubMed: 17186256]

Tower J, Karpen GH, Craig N, Spradling AC. Preferential transposition of Drosophila P elements to nearby chromosomal sites. Genetics 1993;133:347-359. [PubMed: 8382177]

Vagin VV, Klenov MS, Kalmykova AI, Stolyarenko AD, Kotelnikov RN, et al. The RNA interference proteins and vasa locus are involved in the silencing of retrotransposons in the female germline of Drosophila melanogaster. RNA Biol 2004;1:54-58. [PubMed: 17194939]

Vagin VV, Sigova A, Li C, Seitz H, Gvozdev V, et al. A distinct small RNA pathway silences selfish genetic elements in the germline. Science 2006;313:320-324. [PubMed: 16809489]

Villasante A, Abad JP, Planello R, Mendez-Lago M, Celniker SE, et al. Drosophila telomeric retrotransposons derived from an ancestral element that was recruited to replace telomerase. Genome Research 2007;17:1909-1918. [PubMed: 17989257]

Walter MF, Biessmann MR, Benitez C, Török T, Mason JM, et al. Effects of telomere length in Drosophila melanogaster on life span, fecundity, and fertility. Chromosoma 2007;116:41-51. [PubMed: 17089138]

Walter MF, Jang C, Kasravi B, Donath J, Mechler BM, et al. DNA organization and polymorphism of a wild-type Drosophila telomere region. Chromosoma 1995;104:229-241. [PubMed: 8565699]

Watson JD. Origin of concatameric T7 DNA. Nature New Biol 1972;239:197-201. [PubMed: 4507727]

Yin H, Lin HF. An epigenetic activation role of Piwi and a Piwi-associated piRNA in Drosophila melanogaster. Nature 2007;450:304-U316. [PubMed: 17952056] 
A

HeT-A, 6 kb

GAG ORF (3' UTR AAAAAA

TAHRE, $10 \mathrm{~kb}$

GAG ORF ( RT ORF ( 3' UTR AAAAAA

TART, 11-13 kb

GAG ORF ( RT ORF ( 3' UTR AAAAAA

B

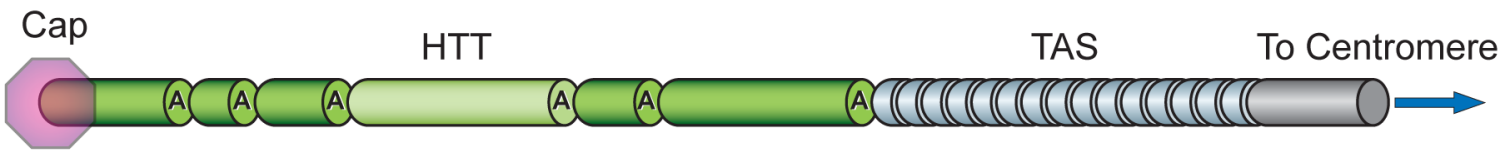

Fig. 1.

(A) Telomeric non-LTR retrotransposons. The GAG and RT open reading frames are indicated. All three elements carry long $3^{\prime}$ UTRs of at least $3 \mathrm{~kb}$. The $3^{\prime}$ oligo(A) tail used to attach to chromosome ends is indicated by AAAAAA. (B) Drosophila telomere structure. Starting at the distal end, the chromosome carries a protein complex that binds to the end independently of DNA sequence and stabilizes the terminus. Most telomeres include a tandem mixed array of variably 5' truncated retrotransposons. The " $\mathrm{A}$ " at each junction indicates the 3 ' oligo(A) tail. Proximal to the retrotransposons is a complex subterminal repeat array, termed telomere associated sequence (TAS), indicated by repeated disks, followed by unique sequence chromosomal DNA. 


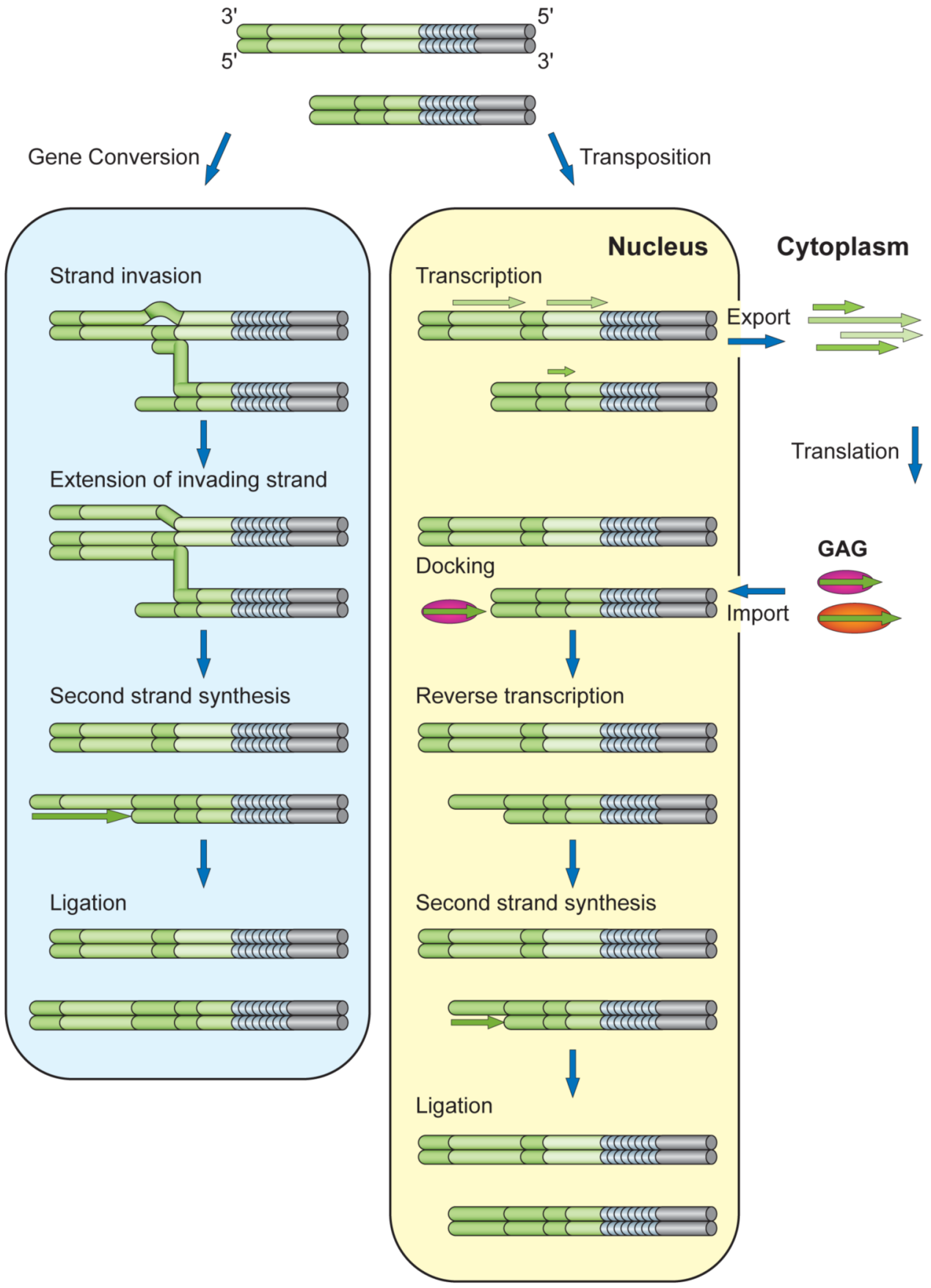

Fig. 2.

Two modes of Drosophila telomere elongation. Gene conversion, on the left, proposes that the 3' strand of a chromosome end invades another chromosome, possibly a sister or homologue. The invading strand is extended using the host sequence as a template, then is used as a template in second strand synthesis. Ligation of the newly replicated fragment results in an extended chromosome. Transposition, on the right, proposes that transcripts are generated from a telomeric retrotransposon using promoter activity located in the $3^{\prime}$ UTR of an upstream HeT$A$ or TAHRE element. The transcripts leave the nucleus to serve as mRNA for the elementencoded GAG-like protein and possibly reverse transcriptase (ovals). GAG-like proteins bind the RNA and facilitate re-entry into the nucleus. After docking to a chromosome end, perhaps 
mediated by a protein-protein interaction between the GAG-like protein and the chromosome cap, a reverse transcriptase uses the free $3^{\prime}$ hydroxyl group at the chromosome end as primer to copy the RNA intermediate into the first DNA strand. Second strand synthesis completes the addition of a new retrotransposon. While it is possible that HTT transcripts that have not left the nucleus may also be used a templates for reverse transcription at the chromosome ends, sequence analyses of very recently transposed $\mathrm{HeT}$-A elements and of several in native telomeric arrays suggest that there is a selection for the incorporation of elements with a functional GAG-like ORF. 

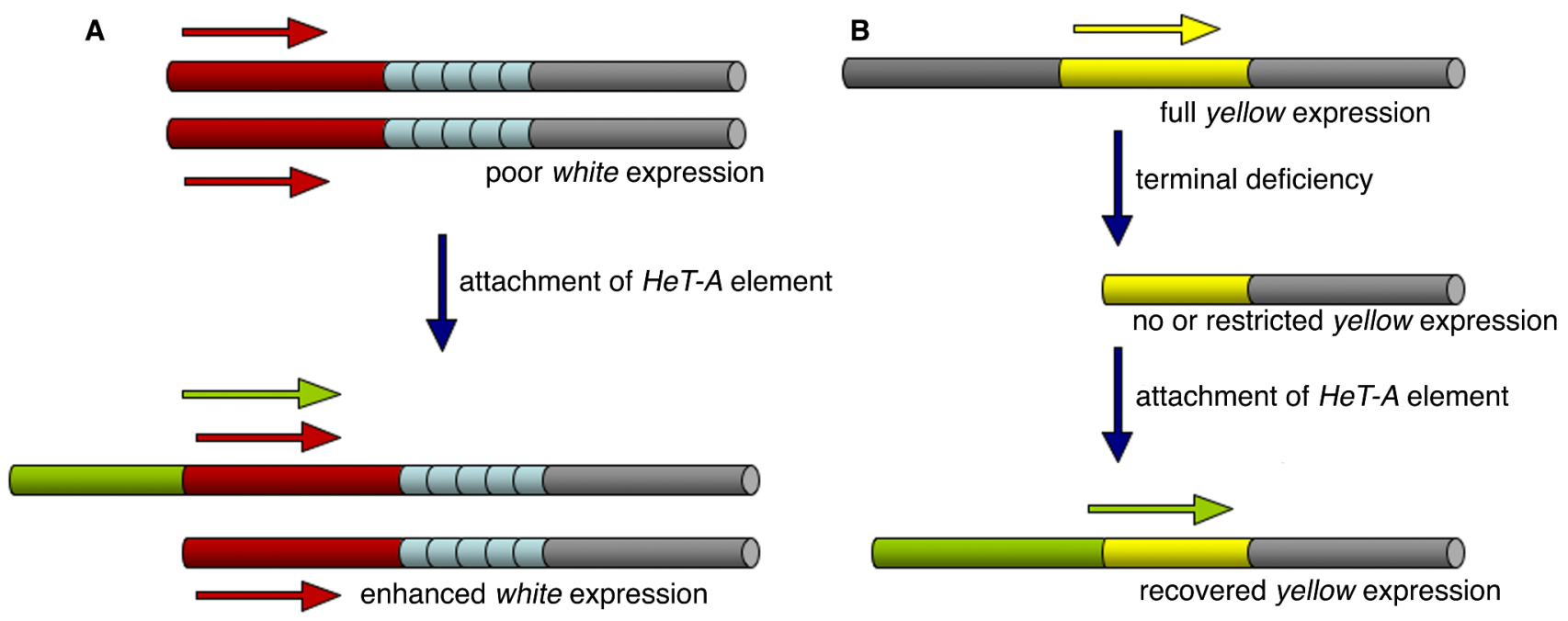

Fig. 3.

Two representative genetic assays for transposition onto chromosome ends. (A) $P\left\{w^{\text {var }}\right.$ ) is a $P$ element containing a complete white $(w)$ gene that has inserted into TAS of the left arm of chromosome 2 associated with loss of the HTT array and part of TAS distal to the insertion (Golubovsky et al., 2001). Some, but not all, variants have some HTT sequence at the end of the chromosome. Those variants with little or no HTT show pale to light orange eyes, depending on the length of the $w$ sequence remaining. Transposition of a $H e T-A$ to the chromosome end allows the HeT-A 3' promoter to initiate transcription into the $w$ gene. This can be seen as a change increased pigment in the eye. (B) The yellow $(y)$ gene is normally located $250 \mathrm{~kb}$ from the $X \mathrm{~L}$ TAS. Terminal deficiencies can be induced that bring the end of the chromosome close to the $y$ promoter and inactivate transcription (Kahn et al., 2000). Transposition of a HeT-A element to the chromosome end allows read-through transcription from the $H e T-A$ promoter into the $y$ gene, resulting in a change in the color of the cuticle and wings. Many of the assays for HTT addition upstream of $y$ at the chromosome end incorporate the $y a c$ deficiency that removes the $y$ gene from the homologous chromosome. Thus, there is only one $y$ gene present. In the presence of a full length homologue in which the $y$ gene is complete, gene conversion can transfer upstream $y$ sequences to the truncated chromosome, as in Figure 2. 